\title{
Bestimmung von Protein im Liquor: Vergleich der Coomassie-Blau-Methode mit dem Cu-Folin-Differentialtest
}

\author{
Von R. Schnell, Silvia Jegge und H. P. Rieder \\ Klinisch-chemisches Laboratorium, Kantonsspital Basel
}

(Eingegangen am 28. Mai/22. Oktober 1982)

Zusammenfassung: Ein Vergleich der Impräzision ,,in der Serie“ und ,von Tag zu Tag“ bei Bestimmungen an Kontrollproben und 96 zufällig verfügbaren Liquorproben des Routinelaboratoriums ergibt, daß der $\mathrm{Cu}$ Folin-Differentialtest und die Coomassie-Brilliantblau-Methode von Bio-Rad zur Bestimmung des Totalproteingehaltes im Liquor etwa gleichwertig sind. Möglicherweise auftretende Probleme bei sehr niedrigen Proteinkonzentrationen im Liquor oder Urin sowie Einflüsse von in der neurologischen Praxis gebräuchlichen Medikamenten werden vorgestellt und diskutiert.

Determination of protein in cerebrospinal fluid:

Comparison of the Coomassie-Blue method and the $\mathrm{Cu}$-Folin differential test

Summary: Comparison of within-run and between-day imprecision of protein assays using CSF controls as well as 96 random CSF samples from the routine laboratory shows that the $\mathrm{Cu}$-Folin differential test and the Coomassie-Brilliant-Blue method from Bio-Rad are equally suited to total protein determination in cerebrospinal fluid. Problems possibly arising with very low concentrations of CSF or urine protein and the influence of some drugs often used in the neurological clinic are presented and discussed.

\section{Einführung}

Nachdem wir in früheren eigenen Versuchen mit Coomassieblau, später dann mit ersten käuflichen Reagenzien keine genügende Farbkonstanz zur sicheren Erfassung der Proteinkonzentrationen erzielen konnten, liegt heute nun eine Reagenzienformulierung der Firma ECS-Bio-Rad vor, mit welcher bessere Ergebnisse erhalten werden.

1976 hat Bradford (1) diese Methode zur Bestimmung kleinster Proteinmengen vorgeschlagen. In = zwischen haben sich mehrere Autoren (2-7) erfolgreich mit deren Verbesserung und Anwendung zur Bestimmung von Liquor- und Urinprotein befaßt. Andere (8) sind von den Eigenschaften und der $\mathrm{Zu}$ verlässigkeit dieses Farbtestes nicht befriedigt.

Wie der nachfolgende Vergleich von 96 Liquorproben und zugehörigen Qualitätskontrollen zeigt, gibt die Bestimmung mit der Coomassie-Brilliant-BlauMethode von Bio-Rad weitgehend gleiche Werte des Totalproteins wie der in unserem Liquor-Laboratorium seit bald zwei Jahrzehnten übliche $\mathrm{Cu}-\mathrm{Fo}$ lin-Differentialtest nach Rieder $(10,11)$.

\section{Methoden \\ Bio-Rad-Test mit Coomassie-Brilliant-Blau}

Proteine bilden mit Coomassie-Brilliant-Blau G250 in saurer Lösung sofort einen Komplex, wobei das Absorptionsmaximum des Farbstoffes von 465 auf $595 \mathrm{~nm}$ verschoben wird. Der Absorptionsgewinn bei $595 \mathrm{~nm}$ ist der Menge des gebundenen Proteins bis zu etwa $1000 \mathrm{mg} / \mathrm{l}$ proportional.

Als Standard dient neuerdings eine Proteinmischung von $0,7 \mathrm{Ge}-$ wichtsteilen humanem Albumin und 0,3 Gewichtsteilen humanem $\gamma$-Globulin (also nicht Albumin allein und nicht Proteine vom Rind!). Gemäß Anleitung im Firmenprospekt (ECS-Bio- 
Rad 1980 (9)) enthält nach vorgeschriebener Verdünnung die Reagenziengebrauchslösung im Liter:

$100 \mathrm{mg}$ Coomassie-Brilliant-Blau G250

0.05 Volumenteile Methanol $18,4 \mathrm{~mol} / \mathrm{l}(95 \%)$

0,10 Volumentcile $o$-Phosphorsäure $15 \mathrm{~mol} / \mathrm{l}(85 \%)$.

100 ul Probe und $5 \mathrm{ml}$ Reagens werden $10 \mathrm{~min}$ nach sorgfältiger Mischung bei $595 \mathrm{~nm}$ abgelesen (9). Die Referenzwerte für Lumballiquoren werden von verschiedenen Autoren etwas unterschiedlich, teils mit $100-600 \mathrm{mg} / \mathrm{l}(2)$, teils mit $90-460 \mathrm{mg} / \mathrm{l}(4)$ oder $150-450 \mathrm{mg} / \mathrm{l}(9)$ angegeben.

\section{Cu-Folin-Differentialtest}

Dieser beruht auf der Lowryschen Cu-Folin-Reaktion in der erweiterten Modifikation von Rieder $(10,11)$. Vorerst handelt es sich um eine 2stufige Reaktion, deren zweiter Schritt durch Fremdeinflüsse (vor allem phenolische Substanzen) gestört werden kann:

(1) $\mathrm{Cu}^{2+}+\mathrm{SO}_{4}^{2-}+$ Protein $\rightarrow$ [Cu-Protein]-Komplex

(2) Phosphormolybdän(VI)-I wolframsäure

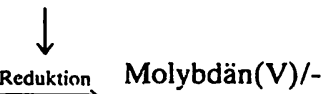

(Folin-Reagens)

wolfram(V)-blau

Das Prinzip des Differentialtests besteht darin, daß der Ansatz $(0,1 \mathrm{ml}$ Probe $+5,5 \mathrm{ml}$ Reagens) stets im Doppel ausgeführt wird, einmal mit Zusatz des $\mathrm{Cu}^{\mathrm{II}}$-Salzes und einmal ohne. Im ersten Ansatz reagieren alle Farbgeber zum intensiven, bei $750 \mathrm{~nm}$ optimal meßbaren Molybdänblau, im zweiten Ansatz (ohne $\mathrm{Cu}$ ) nur die möglichen Fremdsubstanzen in der Sekundäreaktion (z.B. gewisse Medikamente). Bildet man durch Subtraktion der zweiten von der ersten Messung die Differenz der identischen Proben, so erhält man den Farbanteil des reinen Proteins (Primärreaktion). Dieser kann anhand einer entsprechend behandelten Standardlösung ausgerechnet werden.

Als Standard dient auch in dieser Methode seit jeher eine Proteinmischung, und zwar 0,6 Gewichtsteile humanes Albumin (Behring) und 0,4 Gewichtsteile humanes $\gamma$-Globulin (Behring), da der durchschnittliche, mittels Agarelektrophorese nach Wieme (17) ermittelte Albuminanteil unserer Referenzpersonen um 0,63 liegt, derjenige von Patienten oft auch niedriger. Die Differentialmethode umfaßt einen Bereich von 0-1500 mg/l; da die erhaltene Standardkurve eine ganz leichte Biegung aufweist, interpolieren wir die gemessenen Absorptionen zur Umrechnung in Konzentrationen abschnittweise mit Hilfe eines Tischcomputers (15).

Die Referenzwerte für Lumballiquoren liegen zwischen 180-410 $\mathrm{mg} / \mathrm{l}$, diejenigen für suboccipitale Liquoren um $17 \%$ tiefer (15).

\section{Resultate}

\section{Präzision der Methoden}

Die berechneten Kenngrößen sind in Tabelle $1 \mathrm{zu}$ sammengestellt. Aufgrund der relativ großen Zahl vergleichbarer Werte kommen wir : zur UUberzeugung, da $\beta$ die beiden Methoden hinsichtlich ihrer Präzision in der Serie und von Tag zu Tag nicht wesentlich voneinander abweichen.

Hingegen können wir die Firmenangabe (9), daß der Coomassie-Brillant-Blau-Test viermal empfindlicher sein soll als die Lowry-Reaktion, nicht bestätigen, wenn wir auf unsere Modifikation dieses Tests $(10,11)$ abstellen. Aus dem Vergleich des in beiden Methoden verwendeten Fisher-Control-Liquors geht hervor, daß dieser in gleicher Endverdünnung des Reaktionsvolumens $(5,1 \mathrm{ml})$ in der primären $\mathrm{Cu}$ Folin-Reaktion eine Absorption von 0,219, mit Coomassieblau eine solche von 0,365 ergibt, daß der Empfindlichkeitsgewinn mit dem Coomassie-Brillant-Blau-Test also bestenfalls einen Betrag zwischen 1,5-2 erreicht (hier 1,7).

\section{Liquorproteinbestimmung}

96 Proben einer Šerie konsekutiv im Routinelabor eintreffender Liquoren, bei denen genügend Material zur Verfügung stand, wurden gleichzeitig mittels beider Methoden bestimmt. In den vorliegenden Vergleich einbezogen wurden die effektiv gemessenen Konzentrationen, ohne allfällige Aufrechnungen mit Verdünnungsfaktoren (5 Fälle) zu berücksichtigen.

Von früheren Untersuchungen her ist bekannt (10, 11), daß der $\mathrm{Cu}$-Folin-Differentialtest auf Globuline etwas stärker reagiert als auf Albumine. Für labor= diagnostische Zwecke erachteten wir dies als Vorteil, sind doch die meisten pathologischen Veränderungen mit einer Vermehrung von Globulinen verbunden, welche dadurch umso rạscher angezeigt

Tab. 1. Präzision des Cu-Folin-Differentialtests und der Coomassie-Brilliant-Blau G250-Methode.

\begin{tabular}{|c|c|c|c|c|c|c|}
\hline \multirow{2}{*}{$\begin{array}{l}\text { Proben und Proteinkonzentration*) } \\
(\mathrm{mg} / \mathrm{l})\end{array}$} & \multicolumn{3}{|c|}{ Cu-Folin-Differentialtest } & \multicolumn{2}{|c|}{ Coomassieblau-Methode } & \multirow{2}{*}{$\begin{array}{l}\cdots \\
\text { VK } \\
(\%)\end{array}$} \\
\hline & $\mathrm{N}$ & $\begin{array}{l}\bar{x} \pm s \\
(\mathrm{mg} / \mathrm{l})\end{array}$ & $\begin{array}{l}\text { VK } \\
(\%)\end{array}$ & $\mathrm{N}$ & $\begin{array}{l}\bar{x} \pm s \\
(\mathrm{mg} / \mathrm{l})\end{array}$ & \\
\hline \multicolumn{7}{|l|}{ In der Serie } \\
\hline $\begin{array}{l}\text { Albumin-Kontrolle (620) } \\
\text { Fisher-Control (410) } \\
\text { Bio-Rad-Kontrolle (850) } \\
\text { Von Tag zu Tag }\end{array}$ & $\begin{array}{l}88 \\
43 \\
-\end{array}$ & $\begin{array}{l}632 \pm 10,6 \\
392 \pm 9,4 \\
-\end{array}$ & $\begin{array}{l}1,67 \\
2,39 \\
-\end{array}$ & $\begin{array}{l}- \\
40 \\
40\end{array}$ & $\begin{array}{l}- \\
400 \pm 11 ; 0 \\
787 \pm 19,5\end{array}$ & $\begin{array}{l}- \\
2,75 \\
2.47\end{array}$ \\
\hline $\begin{array}{l}\text { Albumin-Kontrolle (620) } \\
\text { Fisher-Control (410) } \\
\text { Bio-Rad-Kontrolle (850) }\end{array}$ & $\begin{array}{l}90 \\
86 \\
-\end{array}$ & $\begin{array}{l}637 \pm 21,2 \\
400 \pm 12,4 \\
-\end{array}$ & $\begin{array}{l}3,32 \\
3,09 \\
-\end{array}$ & $\begin{array}{l}- \\
80 \\
80\end{array}$ & $\begin{array}{l}- \\
399 \pm 12,5 \\
787 \pm 17,1\end{array}$ & $\begin{array}{l}- \\
3,13 \\
2,17\end{array}$ \\
\hline
\end{tabular}


wird. Für Vergleichsuntersuchungen können daraus jedoch Abweichungen resultieren, wenn die Coomassie-Brilliant-Blau-Methode nicht oder im umgekehrten Sinn dem Albumin/Globulin-Verhältnis unterworfen ist $(3,8) .2$ der 96 Probenwerte fallen bei der Korrelation der Ergebnisse als Ausreißer auf (Test for Outliers nach F. E. Grubbs (12), OlivettiProgramm Nr. 9200101). Da wir von beiden Patienten keine Liquorelektrophorese besitzen, anhand welcher wir Albumin-Globulin-Veränderungen hätten beurteilen können und kein Liquor zur Wiederholung der Tests übrigblieb, sind diese zwei Werte aus der nachfolgenden Statistik ausgeschlossen. Abbildung 1 gibt die Korrelation und Regression der Vergleichsbestimmungen wieder. Mit $\mathbf{r}=0,981$ ( $\mathrm{F}$ $=2321,3)$, resp. $r=0,984(F=2793,2)$ im logarithmischen Maßstab, ergibt sich eine sehr gute Korrelation und eine Regressionsgerade, die nahezu mit der Winkelhalbierenden zusammenfällt. Die Meßwerte des Patientenkollektivs lauten für die CoomassieBrilliant-Blau-Methode

$\overline{\mathrm{x}} \pm \mathrm{s}=512 \pm 274 \mathrm{mg} / \mathrm{l}(\log =2,661 \pm 0,198)$

und für den $\mathrm{Cu}$-Folin-Differentialtest

$\overline{\mathrm{y}} \pm \mathrm{s}=505 \pm 272 \mathrm{mg} / \mathrm{l}(\log =2,652 \pm 0,205)$.

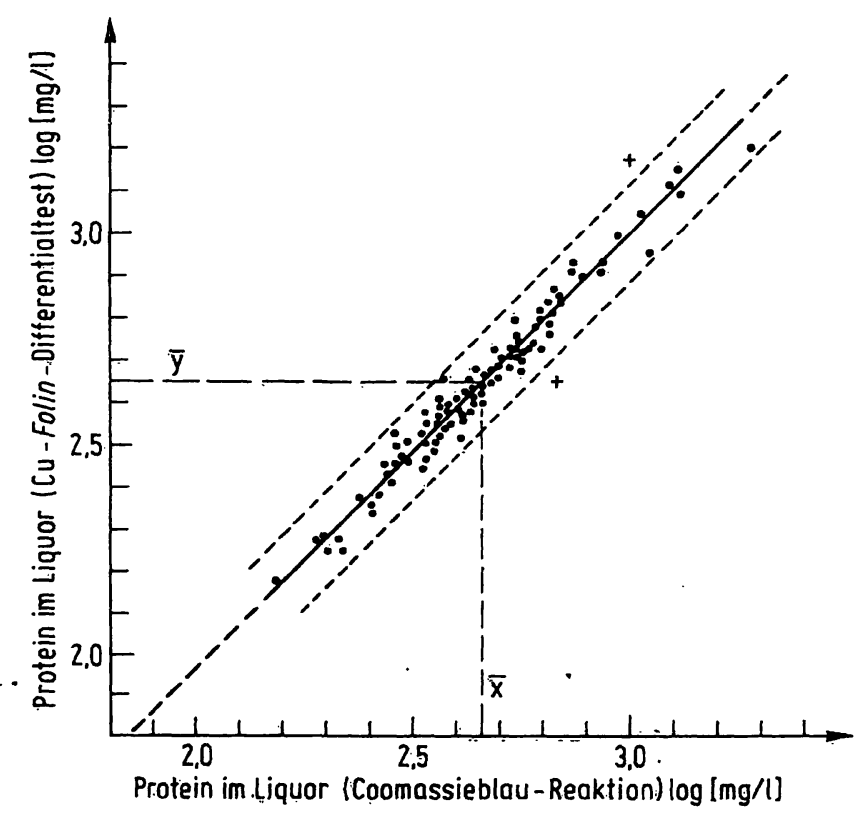

Abb. 1. Vergleichende Totalproteinbestimmung von 96 Liquoren mittels Coomassieblau-Reaktion und Cu-Folin-Differentialtest.

$+=$ zwei nicht wiederholbare Ausreißer (Materialmangel)

... = dreifacher Standardfehler der Residuen $\left(s_{y . x}\right)$

Regression (logarithmisch):

$y=-0,111+1,038 x$

$r=0,984$
Da es sich um ein willkürliches Probenkollektiv mit teilweise pathologischen Werten handelt, weist dieses keine symmetrische Verteilung der Werthöhen auf. Erst durch Logarithmieren der Werte wird eine nahezu normale Verteilung erhalten. Der statistische Vergleich der beiden Methoden, bei welchem wir die Empfehlungen von Haeckel $(13,14)$ zur Berechnung der multivariaten Abhängigkeiten beachteten, erfolgte daher an den logarithmierten Werten. Die so erhaltenen Ergebnisse sind in Tabelle 2 zusammengestellt.

Tab. 2. Steigung und statistische Signifikanz (Probenwerte in $\mathrm{mg} / \mathrm{l}$, logarithmisch, $\mathrm{n}=94$ ).

\begin{tabular}{|c|c|c|c|}
\hline $\begin{array}{l}\text { Methoden und } \\
\text { Proteinkonzentration } \\
(\mathrm{mg} / \mathrm{l}) \text { : }\end{array}$ & $\begin{array}{l}\text { Coomassie- } \\
\text { Brilliant- } \\
\text { Blau-Test }\end{array}$ & & $\begin{array}{l}\text { Cu-Folin- } \\
\text { Differen- } \\
\text { tialtest }\end{array}$ \\
\hline Mittelwerte & $\bar{x}=2,6613$ & & $\bar{y}=2,6525$ \\
\hline Differenz $\bar{x}-\bar{y}$ & $\overline{\mathrm{d}}=$ & 0,0088 & \\
\hline $\begin{array}{l}\text { Signifikanz } \\
\text { (gepaarter t-Test) }\end{array}$ & $t=$ & 2,31 & $(P \sim 0,02)$ \\
\hline $\begin{array}{l}\text { Standardfehler } \\
\text { der Residuen }\end{array}$ & $s_{y, x}=$ & 0,0262 & \\
\hline $\begin{array}{l}\text { Steigung der } \\
\text { standardisierten } \\
\text { Hauptkomponente (b) }\end{array}$ & $\mathrm{b}=$ & 1,038 & \\
\hline Interzept (a) & \multicolumn{3}{|c|}{$a=-0,1111$} \\
\hline $\begin{array}{l}\text { Unterschied } \\
\text { der Steigung von } 1,00 \\
\text { (t-Test nach l.c. }(14) \text { ) }\end{array}$ & \multicolumn{2}{|c|}{$t=-2,02$} & $(P \sim 0,05)$ \\
\hline
\end{tabular}

kursiv $=$ Kenngrößen der Signifikanzrechnung

\section{Medikamenteinflüsse}

Eine Auswahl der in unserer neurologischen Klinik am häufigsten verwendeten Medikamente wurde modellmäßig auf ihre Reaktionsfähigkeit in beiden Methoden überprüft. Zum weiteren Vergleich ist zudem noch die einfache $\mathrm{Cu}$-Folin-Methode (ohne Differentialansatz) in der zusammenfassenden Tabelle 3 angegeben. Die Erfassungsgrenze $(2 \times S D)$, ermittelt aus 19 Doppelbestimmungen von Liquoren mit relativ niedrigen Proteinkonzentrationen $(<300$ $\mathrm{mg} / \mathrm{l})$, beträgt für die Coomassie-Brilliant-Blau-Methode $15,4 \mathrm{mg} / \mathrm{l}$, für den Cu-Folin-Differentialtest $14,0 \mathrm{mg} / \mathrm{l}$. Werte unter $15 \mathrm{mg} / \mathrm{l}$ sind deshalb unzuverlässig und könñten ebensogut gleich Null sein.

Die im Test verwendeten Konzentrationen sind theoretisch; sie würden vielleicht etwa der anfänglichen Maximaldosis im Serum entsprechen. Natürlich wird im Liquor immer nur ein meist minimaler Bruchteil dieser Medikamentkonzentration erreicht. Umso geringfügiger werden dann die zu erwartenden Störungen sein. 
Tab. 3. Fragliche Medikamenteinflüsse bei der Totalproteinbestinmung: Vergleich des Cu-Folin-Differentialtests mit der Coomassieblau-Methode von Bio Rad.

\begin{tabular}{|c|c|c|c|c|c|}
\hline \multirow[t]{2}{*}{ Medikament } & \multirow{2}{*}{\multicolumn{2}{|c|}{$\begin{array}{l}\text { Klinisch appli- } \\
\text { zierte Höchst- } \\
\text { konzentration } \\
\text { bei Annahme } \\
\text { eines Verdün- } \\
\text { nungsvolu- } \\
\text { mens von } 31^{*} \text { ) }\end{array}$}} & \multicolumn{3}{|c|}{$\begin{array}{l}\text { Gemessene Protein- } \\
\text { Äquivalente in } \mathrm{mg} / \mathrm{l}^{* *} \text { ) }\end{array}$} \\
\hline & & & $\begin{array}{l}\mathrm{Cu}-\mathrm{Fo} \\
\text { Reakt } \\
\text { ein- } \\
\text { fach }\end{array}$ & $\begin{array}{l}\text { lin- } \\
\text { on: } \\
\text { Diffe- } \\
\text { ren- } \\
\text { tialtest }\end{array}$ & $\begin{array}{l}\text { Coo- } \\
\text { massie- } \\
\text { blau- } \\
\text { Me- } \\
\text { thode }\end{array}$ \\
\hline \multicolumn{6}{|l|}{ Carbasalat-Calcium } \\
\hline $\begin{array}{l}\text { Diclofenac-Natrium } \\
\text { (Voltaren) }\end{array}$ & 33 & $\mathrm{mg} / \mathrm{l}$ & 33 & 10 & 15 \\
\hline Indometacin (Indocid) & 50 & $\mathrm{mg} / \mathrm{l}$ & 2 & 0 & 16 \\
\hline Baclofen (Lioresal) & 33 & $\mathrm{mg} / \mathrm{l}$ & 6 & 6 & 21 \\
\hline Prednisolon & 330 & $\mathrm{mg} / \mathrm{l}$ & 13 & 0 & 13 \\
\hline $\begin{array}{l}\text { Valproinsäure } \\
\text { (Depakine) }\end{array}$ & 330 & $\mathrm{mg} / \mathrm{l}$ & 0 & 0 & 31 \\
\hline $\begin{array}{l}\text { Carbamazepin } \\
\text { (Tegretol) }\end{array}$ & 300 & $\mathrm{mg} / \mathrm{l}$ & 0 & 0 & 20 \\
\hline Phenobarbital & 66 & $\mathrm{mg} / \mathrm{l}$ & 4 & 4 & 18 \\
\hline Phenytoin & 133 & $\mathrm{mg} / \mathrm{l}$ & 0 & 0 & 13 \\
\hline Digoxin & & $\mathrm{mg} / \mathrm{l}$ & 7 & 8 & 15 \\
\hline Propanolol (Inderal) & 3 & $\mathrm{mg} / \mathrm{l}$ & 9 & 10 & 15 \\
\hline Haloperidol (Haldol) & 30 & $\mathrm{mg} / \mathrm{l}$ & 4 & 0 & 17 \\
\hline Diazepam (Valium) & 80 & $\mathrm{mg} / \mathrm{l}$ & 0 & 0 & 18 \\
\hline $\begin{array}{l}\text { Chlordiazepoxid } \\
\text { (Librium) }\end{array}$ & 33 & $\mathrm{mg} / \mathrm{l}$ & 6 & 6 & 11 \\
\hline Imipramin (Tofranil) & 50 & $\mathrm{mg} / \mathrm{l}$ & 151 & 0 & 18 \\
\hline Thioridazin (Melleril) & 200 & $\mathrm{mg} / \mathrm{l}$ & 103 & 0 & 53 \\
\hline $\begin{array}{l}\text { Phenprocoumon } \\
\text { (Marcoumar) }\end{array}$ & 6 & $\mathrm{mg} / \mathrm{l}$ & 0 & 0 & 14 \\
\hline Mannit & 33 & $\mathrm{mg} / \mathrm{l}$ & 308 & 390 & 16 \\
\hline Penicillin & $\begin{array}{l}1000 \\
\text { intrat } \\
\text { d.h. } p\end{array}$ & $\begin{array}{l}0 \mathrm{IE} \\
\text { hekal } \\
\text { ro } 130 \mathrm{ml}\end{array}$ & 39 & 41 & 20 \\
\hline $\begin{array}{l}\text { Metrizamid } \\
\text { (Amipaque) }\end{array}$ & {$[\mathrm{I}]=1$} & $\left.100 \mathrm{~g} / \mathrm{l}^{* * *}\right)$ & 2 & 2 & 16 \\
\hline Lidocain (Xylocain) & 100 & $\mathrm{mg} / \mathrm{l}$ & 22 & 12 & 1 \\
\hline
\end{tabular}

*) mit Modell-Lösungen dieser Konzentration, gelöst in isoton. $\mathrm{NaCl}$ (bei Säuren unter Zusatz von etwas $\mathrm{NaOH}$ ) wurden die Vergleichsbestimmungen ausgeführt (sofern nicht vollständig löslich = gesättigte Lösung).

**) Erfassungsgrenzen: siehe Text.

***) Iodkonzentration pro 1 Kontrastmittellösung.

\section{Diskussion}

Die vorliegende Untersuchung zeigt, daß die beiden Methoden etwa die gleiche Präzision aufweisen. Der direkte Vergleich an Liquoren neurologischer $\mathrm{Pa}$ tienten führt ebenfalls zum Eindruck annähernder Gleichwertigkeit, wenn man davon absieht, daß schwach signifikant höhere Werte bei der Coomassieblau-Methode zumindest gruppenstatistisch nachgewiesen werden können.
Im klinischen Einzelfall erscheint dieser minime Unterschied allerdings belanglos, vor allem deshalb, weil bei Liquoren mit zunehmend pathologischen Werten die Úbereinstimmung besser wird. Dieser Umstand ist auch aus dem Verlauf der Regressionsgeraden (Abb. 1) ersichtlich; während diese im Bereich um $1000 \mathrm{mg} / \mathrm{l}$ die Winkelhalbierende schneidet - also beide Methoden identische Werte geben - weist die Coomassie-Brilliant-Blau-Methode im Bereich sehr kleiner Liquorproteinwerte eine Tendenz zu höheren Werten auf. Die Regressionssgerade geht nicht durch Null, wodurch die Richtigkeit des Analysenwertes sehr niedriger Konzentrationen beeinträchtigt wird. Man darf daraus wohl die Vermutung ableiten, da $\beta$ die Standardgerade im untersten Teil nicht linear verläuft. Dieser Umstand ist vor allem bei Arbeiten mit Kinderliquoreñ, deren Proteingehalte häufig niedriger als bei Erwachsenen liegen, wichtig und zu beachten. Er dürfte mit ein Grund sein, daß bei den Medikamentversuchen alle Coomassie-Brilliant-Blau-Werte systematisch um $10-15 \mathrm{mg} / \mathrm{l}$ angehoben sind.

Außer diesem Nachteil muß weiterhin berücksichtigt werden, daß der starken Haftfähigkeit des Coomassie-Farbstoffes wegen eigentlich nur mit Wegwerfküvetten von Hand gearbeitet werden sollte oder $\mathrm{da} \beta$ - z.B. mit Methanol - laufend zwischengespült werden muß, was beides vermehrten Z̄eitaufwand bedeutet. Davon abgesehen ist die CoomassieblauMethode an sich relativ rasch und stellt etwas geringere Ansprüche an den Berechnungsaufwand. Für die Proteinbestimmung im Urin ist sie der $\mathrm{Cu}$ Folin-Methode sogar vorzuzièhen, weil letztere durch die Vielzahl von Fremdsubstanzen im sehr variablen Milieu des Urins allzu stark gestört wird. Eine erhöhte Ausflockungstendenz des Coomassieblau-Farbstoffes kann allerdings auch hier die Analyse beeinträchtigen, wenn nicht rasch gearbeitet und die Gebrauchslösung häufig filtriert wird.

Aufgrund der weitgehend identischen Ergebnisse beider Methoden im Liquor-Vergleich und unter Berụ̈cksichtigung der tendenziell etwas höheren Werte der Coomassie-Brilliant-Blau-Methode bei geringem Totalproteingehalt müßten wir in Anlëhnung an unsere eigenen Werte des klinișch-normalen Referenzkollektivs (15) für die CoomassieblauMethode die folgenden "Referenzbereiche" des Lumballiquors vorscblagen:

Erwachsene 190-420 mg/l,

Kinder $130-400 \mathrm{mg} / \mathrm{l}$ (je nach Alter, vgl. Maurer (16). 
Wenn auch Störungen durch Fremdsubstanzen nie ganz auszuschließen sind, so zeigt doch die Untersuchung der gebräuchlichsten Medikamente, welche in der Neurologischen und Neurochirurgischen Klinik zum Einsatz gelangen, daß von dieser Seite bei beiden Methoden wenig Störeinflüsse zu erwarten sind; einzig Mannit und Penicillin, gegebenenfalls auch Lidocain beim $\mathrm{Cu}$-Folin-Differentialtest sowie Valproinsäure (Depakine) und Thioridazin (Melleril) beim Coomassie-Brilliant-Blau-Test weisen hier etwas kritische Werte auf. Doch dürften solche Konzentrationen im Liquor kaum erreicht werden, mit
Ausnahme des Penicillins, welches direkt intrathekal appliziert wird.

Wägt man alle diese Vor- und Nachteile ab, so darf man feststellen, daß die beiden Methoden hinsichtlich Brauchbarkeit im Routinelabor mehr oder weniger gleichwertig sind und daß es von der spezifischen Fragestellung abhängen wird, für welche der beiden man sich entscheidet, sofern man nicht die sowohl apparativ wie bezüglich Reagenzien bedeutend teureren immunochemischen Analysenmethoden vorzieht.

\section{Literatur}

1. Bradford, M. M. (1976) Anal. Biochem. 72, 248-254.

2. McIntosh, J. C. (1977) Clin. Chem. 23, 1939-1940.

3. Johnson, J. A. \& Lott, J. A. (1978) Clin. Chem. 24, 1931-1933.

4. Pierce, J. \& Suelter, C. H. (1977) Anal. Biochem. 81, $478-480$.

5. Nesbitt, J., Sauer, D. \& Culpepper, R. (1978) J. Amer. Med. Technol. 40, 278-279.

6. Thomas, L., Winckelmann, M., Michaelis, H. C. \& Walb, D. (1981) J. Clin. Chem. Clin. Biochem. 19, 203-208.

7. Miller, N., Mezei, L. M., Gindler, E. M. \& Staley, J. (1979) Clin. Chem. 25, 1071

8. Van Wilgenburg, M. G. M., Werkman, E. M. A., van Gorkom, W. H. \& Soons, J. B. J. (1981) J. Clin. Chem. Clin. Biochem. 19, 301-304.

9. ECS-Bio-Rad Prospekt 1980, Bio-Rad-Laboratories AG, Kanalstraße 17, CH-8152 Glattbrugg.

10. Rieder, H. P. (1966) Klin. Wochenschr. 44, 1036-1046.

11. Rieder, H. P. (1974) in: Clinical Biochemistry, Verlag W. de Gruyter, Berlin. S. 1468-1478.

12. Grubbs, F. E. (1950) Ann. Math. Statist. 21, 27-58; Olivetti Computer-Programm Nr. 9200101.

13. Haeckel, R. (1982) J. Clin. Chem. Clin. Biochem. 20, 107-110.

14. Feldmann, U., Schneider, G., Klinkers, H. \& Haeckel, R. (1981) J. Clin. Chem. Clin. Biochem. 19, 121-137.

15. Jung, P. R., Rieder, H. P. \& Jeltsch, C. (1973) Klin. Wochenschr. 51, 810-816.

16. Maurer, J. \& Rieder, H. P. (1978) Schweiz. Med. Wochenschr. 108, 1854-1860.

17. Rieder, H. P., Jung, P. R. \& Burri, V. (1972) Z. Klin. Chem. Klin. Biochem. J0, 379-384.

\section{Dr. H. P. Rieder}

Leiter des Liquor- und Eiweißlabors

Kantonsspital

CH-4031 Basel 
